\title{
Non-planar data of $\mathcal{N}=4$ SYM
}

\author{
Thiago Fleury ${ }^{a}$ and Raul Pereira ${ }^{b}$ \\ ${ }^{a}$ International Institute of Physics, Federal University of Rio Grande do Norte, \\ Campus Universitário, Lagoa Nova, Natal, RN 59078-970, Brazil \\ ${ }^{b}$ School of Mathematics and Hamilton Mathematics Institute, Trinity College Dublin, \\ College Green, Dublin 2, Ireland \\ E-mail: tsi.fleury@gmail.com, raul@maths.tcd.ie
}

\begin{abstract}
The four-point function of length-two half-BPS operators in $\mathcal{N}=4 \mathrm{SYM}$ receives non-planar corrections starting at four loops. Previous work relied on the analysis of symmetries and logarithmic divergences to fix the integrand up to four constants. In this work, we compute those undetermined coefficients and fix the integrand completely by using the reformulation of $\mathcal{N}=4 \mathrm{SYM}$ in twistor space. The final integrand can be written as a combination of finite conformal integrals and we have used the method of asymptotic expansions to extract non-planar anomalous dimensions and structure constants for twisttwo operators up to spin eight. Some of the results were already known in the literature and we have found agreement with them.
\end{abstract}

KEYwords: 1/N Expansion, Integrable Field Theories, Supersymmetric Gauge Theory

ARXiv EPrint: 1910.09428 


\section{Contents}

1 Introduction 1

1.1 Four-point function of $20^{\prime}$ operators 3

2 Twistors 5

$2.1 \mathcal{N}=4 \mathrm{SYM}$ in twistor space $\quad 5$

$\begin{array}{ll}2.2 & \text { Correlation functions of the stress-tensor multiplet }\end{array}$

$\begin{array}{ll}2.3 \text { Four-loop four-point function } & 10\end{array}$

$\begin{array}{llr}3 & \text { OPE analysis } & 14\end{array}$

$\begin{array}{lll}4 \text { Conclusion } & 17\end{array}$

$\begin{array}{lr}\text { A Conventions } & 19\end{array}$

$\begin{array}{ll}\text { B Examples of fermionic integrations } & 19\end{array}$

\section{Introduction}

The correlation functions of length-two half-BPS operators (also known as 20' operators) in $\mathcal{N}=4$ SYM have been studied extensively in the literature both at weak [1-5] and strong coupling [6-19]. It is well known that the two- and three-point functions of those operators are protected by supersymmetry [7, 20] while higher-point functions receive non-trivial corrections. Each loop order of the correlation function can be computed through the Lagrangian insertion procedure, and because the $20^{\prime}$ operator and the Lagrangian belong to the same supermultiplet, the integrand exhibits a hidden permutation symmetry [3]. Imposing also conformal symmetry and restrictions from logarithmic divergences of the correlator in diverse OPE limits, the planar integrand of the four-point correlator has been fixed up to ten loops $[4,5]$. It is however important to note that knowledge of the integrated correlator is still quite incomplete since conformal integrals are generally not known starting from four loops. Meanwhile, at the non-planar level much less is known. It is easy to see that at one and two loops the non-planar corrections are zero since it is not possible to draw any non-planar Feynman graph at these loop orders. However, the vanishing of the three-loop correction is non-trivial as it results from cancellations of different terms. Finally, at four loops the integrand is known to be a linear combination of four polynomials with constant coefficients. One of the main results of this work is the determination of those four undetermined coefficients. We expect that the methods described below can be adapted to the calculation of other correlators as well.

One of the motivations for computing the four-loop non-planar integrand is that it allows to extract non-planar OPE data by taking a double coincidence limit. In this 
work we have computed both anomalous dimensions and structure constants for twist-two operators up to spin eight. Even at the planar level, where the tools of integrability are more developed, this method is the best way we currently have for obtaining structure constants of unprotected operators at a high loop order [21-24]. In contrast, it was only recently that a direct two-loop perturbative computation of planar structure constants was performed [25, 26]. The double OPE limit of each conformal integral can be taken by using the method of asymptotic expansions described for example in [21,27]. With this method the integration domains are split into distinct regions, which correspond to the different scales of the problem. Effectively, the conformal four-point integrals can be rewritten in terms of twopoint integrals, which are much more tractable. Note that the pseudo-conformal integrals arising in high-loop integrands can also be approximated with this method, as long as one does not assume dependence on cross-ratios. This is however not relevant to this work, as we can write the four-point function in terms of convergent integrals only.

The non-planar anomalous dimensions of twist-two operators up to spin six were also computed in the series of papers [28-30] by a direct Feynman diagrammatic calculation and we have found agreement with them. ${ }^{1}$ It would be very interesting to find a closed expression for the non-planar anomalous dimensions of twist-two operators for any spin. We expect the result to be given in terms of harmonic sums and Riemann zeta values and to obey the principle of uniform transcendentality. Unfortunately the data obtained in this work is not enough to fix the expression for general spin even if one restricts to a smaller basis consisting only of binomial harmonic sums [31]. The knowledge of the non-planar anomalous dimension for generic spin would allow to compute the non-planar cusp anomalous dimension analytically and also take the BFKL limit. These results are important for understanding non-planar integrability or possible formulations of a non-planar quantum spectral curve [32], see [33] for progress in this direction. One should also stress that the non-planar cusp anomalous dimension was computed numerically by studying Sudakov form-factors with a suitable rewriting in terms of uniformly transcendental integrals [34-36].

Another motivation for computing non-planar structure constants is to further the understanding of non-planar integrability. Three-point functions can be computed at the planar level as a product of two integrable hexagon form-factors [37], and it was later understood that higher-point functions can also be decomposed into a weighted product of hexagon form-factors, both at the planar and non-planar level [38-43]. The integrability setup was tested at two loops for long operators and at one loop for short operators such as the $20^{\prime}$. In the case of four-point functions of length-two operators, all the non-planar corrections in the integrability setup come from the stractification procedure described in [41]. More specifically, one embeds the tree-level planar graphs in higher genus surfaces and properly subtracts possible boundary terms. It would be interesting to test the stratification procedure at higher loops (at the moment four loops seems a difficult task) or at least understand why non-trivial non-planar corrections to this correlator first show up at four loops.

\footnotetext{
${ }^{1}$ In fact there is a mismatch for the spin six data. Our coefficient for $\zeta_{3}$ is ten times the one in [30]. Because the mismatch is simple, we believe that there is a typo on the paper [30].
} 
This paper is organized as follows. In the remaining part of the introduction, we review what is known in the literature about four-point functions of $20^{\prime}$ operators up to four loops. In section 2 we introduce the reformulation of $\mathcal{N}=4 \mathrm{SYM}$ in twistor space and explain how to compute correlation functions in that framework. We then describe the strategy used to fix the four-loop non-planar integrand. The OPE analysis of the correlator is performed in section 3, where we present the non-planar OPE data of twist-two low-spin operators. We conclude in section 4 and refer the reader to the appendices for conventions and examples of analytic computations using twistors.

\subsection{Four-point function of $20^{\prime}$ operators}

We will now review some well-known results about correlation functions in $\mathcal{N}=4$ SYM. We will focus our attention on the four-point function of length-two half-BPS operators, which can be written as

$$
\mathcal{O}_{\mathbf{2 0}}\left(x_{i}, y_{i}\right)=y_{i} y_{j} \operatorname{Tr}\left(\Phi^{i} \Phi^{j}\right)\left(x_{i}\right),
$$

with $\Phi^{i}$ the six real scalars of the theory and $y_{i}$ a null polarization vector which projects the operator into the symmetric traceless representation. The correlator admits a double expansion in the effective coupling constant $a=g_{\mathrm{YM}}^{2} N_{c} /\left(4 \pi^{2}\right)$ and in the number of colours $N_{c}$

$$
G_{4}=\left\langle\mathcal{O}_{2 \mathbf{0}^{\prime}}\left(x_{1}, y_{1}\right) \mathcal{O}_{\mathbf{2 0 ^ { \prime }}}\left(x_{2}, y_{2}\right) \mathcal{O}_{\mathbf{2 0 ^ { \prime }}}\left(x_{3}, y_{3}\right) \mathcal{O}_{\mathbf{2 0 ^ { \prime }}}\left(x_{4}, y_{4}\right)\right\rangle=\sum_{\ell \geq 0} \sum_{g \geq 0} \frac{a^{\ell}}{N_{c}^{2 g}} G_{4}^{(g, \ell)}\left(x_{i}, y_{i}\right)
$$

Starting at one loop, $\mathcal{N}=4$ superconformal symmetry [44] implies that at any order in $N_{c}$ the correlator $G_{4}^{(g, \ell)}$ factorizes in the following way

$$
G_{4}^{(g, \ell>0)}\left(x_{i}, y_{i}\right)=2\left(N_{c}^{2}-1\right) \times R\left(x_{i}, y_{i}\right) \times F^{(g, \ell)}\left(x_{i}\right),
$$

where all depence on the polarization vectors of the external operators is encoded in the $R$ factor

$$
\begin{aligned}
& R\left(x_{i}, y_{i}\right)=\frac{y_{12}^{4} y_{34}^{4}}{x_{12}^{2} x_{34}^{2}}+\frac{y_{13}^{4} y_{24}^{4}}{x_{13}^{2} x_{24}^{2}}+\frac{y_{14}^{4} y_{23}^{4}}{x_{14}^{2} x_{23}^{2}}+\frac{y_{12}^{2} y_{23}^{2} y_{34}^{2} y_{14}^{2}}{x_{12}^{2} x_{23}^{2} x_{34}^{2} x_{14}^{2}}\left(x_{13}^{2} x_{24}^{2}-x_{12}^{2} x_{34}^{2}-x_{14}^{2} x_{23}^{2}\right) \\
& +\frac{y_{12}^{2} y_{13}^{2} y_{24}^{2} y_{34}^{2}}{x_{12}^{2} x_{13}^{2} x_{24}^{2} x_{34}^{2}}\left(x_{14}^{2} x_{23}^{2}-x_{12}^{2} x_{34}^{2}-x_{13}^{2} x_{24}^{2}\right)+\frac{y_{13}^{2} y_{14}^{2} y_{23}^{2} y_{24}^{2}}{x_{13}^{2} x_{14}^{2} x_{23}^{2} x_{24}^{2}}\left(x_{12}^{2} x_{34}^{2}-x_{14}^{2} x_{23}^{2}-x_{13}^{2} x_{24}^{2}\right) .
\end{aligned}
$$

Notice that all dynamical information is contained in the functions $F^{(g, \ell)}$, which crucially multiply all six $R$-symmetry structures. We can therefore work with a particular choice of polarizations where only $y_{12}$ and $y_{34}$ are non-zero, so that a single term in the $R$ factor survives. This will significantly reduce the number of graphs to be computed, as explained in more detail later in the next section, but one can unambiguously reconstruct the correlator for generic polarizations.

Loop corrections for the correlator can be obtained by the Lagrangian insertion procedure, where the integrand of the $\ell$-loop four-point function is viewed as a Born-level $(4+\ell)$-point function. We can then rewrite the dynamical function as

$$
F^{(g, \ell)}\left(x_{i}\right)=\frac{x_{12}^{2} x_{13}^{2} x_{14}^{2} x_{23}^{2} x_{24}^{2} x_{34}^{2}}{\ell !} \int d^{4} x_{5} \ldots d^{4} x_{4+\ell} f^{(g, \ell)}\left(x_{1}, \ldots, x_{4+\ell}\right),
$$


where the integrand $f^{(g, \ell)}$ carries conformal weight +4 in all external and internal points. Furthermore, an analysis of the possible OPE singularities indicates that the integrand is a rational function which diverges at most as a simple pole in the coincidence limit $x_{i j} \rightarrow 0$, which implies that we can rewrite it as

$$
f^{(g, \ell)}\left(x_{1}, \ldots, x_{4+\ell}\right)=\frac{P^{(g, \ell)}\left(x_{1}, \ldots, x_{4+\ell}\right)}{\prod_{1 \leq i<j \leq 4+\ell} x_{i j}^{2}} .
$$

Finally, $P^{(g, l)}$ is a linear combination of polynomials which have the following properties:

1. It is homogeneous in $x_{i j}^{2}$.

2. It has conformal weight $-(\ell-1)$ at each point.

3. It is invariant under the permutation of all its arguments, i.e. under the group $S_{4+\ell}$.

Property 2 follows from conformal symmetry, while property 3 reflects a hidden permutation symmetry, which follows from the fact that the Lagrangian operator is in the same supermultiplet of the external $\mathbf{2 0}$ ' operators.

At each loop order there is a finite number of polynomials $P_{i}^{(\ell)}$ that satisfy the properties listed above and each $P^{(g, l)}$ is a linear combination of those with constant coefficients. Note that the properties above are independent of $g$, so the basis $P_{i}^{(\ell)}$ which solves these constraints can be used to construct the numerator of the integrand at any order of the genus expansion. At one-, two-, three- and four-loops there are 1, 1, 4 and 32 independent polynomials respectively. We will not write them explicitly in this work so we refer the reader to reference [4]. In order to fix the integrand completely, one only needs to find the coefficient multiplying each polynomial. A powerful method to fix these coefficients is to study the asymptotic behaviour of the correlator either in the Euclidean double shortdistance limit, where both $x_{1} \rightarrow x_{2}$ and $x_{3} \rightarrow x_{4}$, or the Minkowski light-cone limit where $x_{12}^{2}, x_{23}^{2}, x_{34}^{2}, x_{41}^{2} \rightarrow 0$. In these limits the logarithm of the correlator must develop soft logarithmic singularities, which imposes strong constraints on the coefficients. These constraints, together with the conformal Gram determinant relations ${ }^{2}$ were powerful enough to fix the planar four-loop result and to reduce the non-planar corrections at four loops to only four unknown coefficients.

Before writing down the form of the non-planar integrand, let us clarify the classification of the polynomials regarding their planarity. For each homogeneous polynomial $P_{i}^{(\ell)}$ obeying the conformal and permutation symmetries described above, it is possible to associate a graph $f_{i}^{(\ell)}$ via (1.6). Each graph has $4+\ell$ vertices and they are connected by propagators, which are the elements of the denominator left in (1.6) after cancellation with factors from $P_{i}^{(\ell)}$, while the remaining numerator factors plays no role in the $f$-graph. A nice feature of these $f$-graphs is that they are in a sense related to the usual Feynman graphs and we can naturally associate a genus to them in the standard way. More specifically, it was argued in [4] that, apart from the singular one-loop case, the $1 / N_{c}^{2 g}$ correction

\footnotetext{
${ }^{2}$ The Gram determinant condition expresses the fact that in $d$ dimensions there are only $d$ independent vector positions $x^{\mu}$. This constraint can be imposed in a conformally invariant way, leading to a conformal Gram determinant, see appendix B of [4] for details.
} 
to the correlator $G_{4}$ is given by $f$-graphs whose genus is at most $g$. In conclusion, each polynomial $P_{i}^{(\ell>1)}$ is assigned a genus equal to that of its associated $f$-graph and it can only contribute to the integrand $P^{(g, \ell)}$ if the genus obtained does not exceed $g$.

The analysis of the non-planar integrand in [4] showed that corrections to $G_{4}$ first appear at four loops, but the constraints were not sufficient to fix it uniquely. At genus one, the integrand is given up to four undetermined coefficients

$$
P^{(1,4)}\left(x_{1}, \ldots, x_{8}\right)=c_{1} Q_{1}\left(x_{i}\right)+c_{2} Q_{2}\left(x_{i}\right)+c_{3} Q_{3}\left(x_{i}\right)+c_{4} Q_{4}\left(x_{i}\right) .
$$

Each term is a linear combination of the 32 four-loop polynomials $P_{i}^{(4)}$ (see equations (5.9) and (C.1) of [4] for definitions)

$$
Q_{k}\left(x_{1}, \ldots, x_{8}\right)=\sum_{j=1}^{32} q_{k, j} P_{j}^{(4)}\left(x_{1}, \ldots, x_{8}\right),
$$

with

$$
\begin{aligned}
& q_{1}=\left\{0^{26}, 1,0^{5}\right\} \\
& q_{2}=\left\{0,-2,2,-8,0,-6,0^{4}, 2,-2,1,0^{4},-2,-1,1,6,0^{11}\right\}, \\
& q_{3}=\left\{0,-2,-3,4,1,-6,-2,2,-4,0,2,-2,1,1,-1,2,1,0^{15}\right\}, \\
& q_{4}=\left\{-8,-14,10,-8,8,-18,0,3,-4,-4,0,-2,3,4,-2,6,0,-2,-4,4,0,12,2,-2,2,0^{7}\right\} .
\end{aligned}
$$

where the short-hand notation $0^{n}$ corresponds to a list of $n$ zeros.

One of the results of this work is the determination of the coefficients $c_{i}$. We obtained $c_{1}=c_{2}=c_{3}=0$ and $c_{4}=-6$, and in that way we fixed the non-planar integrand at four loops completely. The method we have used relies on the reformulation of $\mathcal{N}=4 \mathrm{SYM}$ in twistor space, which is the subject of the next section.

\section{Twistors}

In this section, we first review how to compute correlation functions of the stress-tensor supermultiplet in $\mathcal{N}=4$ SYM using twistor space, see [45] for further details. One of the advantages of this formalism is that each Feynman diagram in twistor space has manifest $\mathcal{N}=$ 4 superconformal symmetry apart from some reference twistor. Then we explain how the four-loop non-planar calculation was performed for a particular polarization of the external operators. The necessary graphs were generated with the open source program Sage [46].

\section{$2.1 \mathcal{N}=4 \mathrm{SYM}$ in twistor space}

The supertwistor space $[47,48]$ is the complex projective superspace $\mathbb{C P}^{3 \mid 4}$. An element $\mathcal{Z}^{A}$ of this space has four bosonic and four fermionic coordinates and it is defined up to the equivalence relation $\mathcal{Z}^{A} \sim c \mathcal{Z}^{A}$, with $c \in \mathbb{C}^{*}$. These variables are parametrized in the following way

$$
\mathcal{Z}^{A}=\left(\lambda_{\alpha}, \mu^{\dot{\alpha}}, \chi^{a}\right)
$$


with $\alpha, \dot{\alpha}=1,2$ and $\chi^{a}, a=1, \ldots, 4$, the fermionic coordinates. A nice property of these variables is that they transform linearly under the action of all generators of the complexified super conformal group $\operatorname{SL}(4 \mid 4 ; \mathbb{C})$, see for example $[49,50]$ for the explicit form of the generators. In addition, these variables can be related to the usual superspace variables $\left(x^{\alpha \dot{\alpha}}, \theta^{a \alpha}, \bar{\theta}_{\dot{a}}^{\dot{\alpha}}\right)$. We are interested here only in the chiral superspace, i.e. we are going to set all $\bar{\theta}_{\dot{a}}^{\dot{\alpha}}$ to zero, and in this case

$$
\mu^{\dot{\alpha}}=i x^{\dot{\alpha} \beta} \lambda_{\beta}, \quad \chi^{a}=\theta^{a \alpha} \lambda_{\alpha}
$$

These are called incidence relations and they map a point in chiral superspace to a line in supertwistor space.

The first relation in (2.2) can be understood as follows. For simplicity, let us consider the bosonic components of the supertwistors, in which case the complexified conformal group is $\mathrm{SL}(4 ; \mathbb{C})$. The supersymmetric case is a simple generalization. The twistors $Z^{I}=\left(\lambda_{\alpha}, \mu^{\dot{\alpha}}\right)$, with $I=1, \ldots, 4$, transform in the fundamental representation of this bosonic group. We can define a null antisymmetric tensor $X^{I J}$ as (see the appendix A for conventions)

$$
X^{I J}=\left(\begin{array}{cc}
\epsilon_{\alpha \beta} & -i x_{\alpha}^{\dot{\beta}} \\
i x_{\beta}^{\dot{\alpha}} & -x^{2} \epsilon^{\dot{\alpha} \dot{\beta}}
\end{array}\right), \quad X_{I J}=\frac{1}{2} \epsilon_{I J K L} X^{K L}, \quad X_{I J} X^{I J}=0 .
$$

These tensors are also homogeneous, with $X^{I J} \sim c X^{I J}$, and the set of null rays is in correspondence with the original $4 d$ spacetime coordinates $x^{\alpha \dot{\beta}}$. This identification is known as the embedding formalism. Because of the null condition given in (2.3), the matrix $X^{I J}$ has rank two and it can be written in terms of two twistors as

$$
X^{I J}=Z_{1}^{I} Z_{2}^{J}-Z_{1}^{J} Z_{2}^{I} .
$$

As mentioned before, this implies that a spacetime point is mapped to a line in twistor space. The line connects the two twistors $\left\{Z_{1}^{I}, Z_{2}^{J}\right\}$ which have linearly independent values for $\lambda_{\alpha}$ and the component $\mu^{\dot{\alpha}}$ given by $(2.2)$.

It is possible to reformulate $\mathcal{N}=4 \mathrm{SYM}$ in supertwistor space and in that way we gain an alternative method for computing correlation functions of the stress-tensor multiplet. The fields of $\mathcal{N}=4 \mathrm{SYM}$ sit inside a one-form superfield $\mathcal{A}$ living in supertwistor space. Accordingly, the action can be written as a function of this superfield in the following way (see [51, 52] for details)

$$
S_{\mathcal{N}=4}=\int_{\mathbb{C P}^{3 \mid 4}} \mathcal{D}^{3 \mid 4} \mathcal{Z} \wedge \operatorname{Tr}\left(\frac{1}{2} \mathcal{A} \bar{\partial} \mathcal{A}-\frac{1}{3} \mathcal{A}^{3}\right)+g_{\mathrm{YM}}^{2} \int d^{4} x d^{8} \theta L_{\mathrm{int}}(x, \theta) .
$$

In order to perform calculations, it is convenient to choose a gauge in which a component of the superfield $\mathcal{A}$ vanishes in the direction of a reference twistor $\mathcal{Z}_{\diamond}$, so that the kinetic term becomes quadratic and the interaction term simplifies to

$$
L_{\text {int }}(x, \theta)=-\sum_{k \geq 2} \frac{1}{k} \int D \sigma_{1} \ldots D \sigma_{k} \frac{\operatorname{Tr}\left[\mathcal{A}\left(\mathcal{Z}\left(\sigma_{1}\right)\right) \ldots \mathcal{A}\left(\mathcal{Z}\left(\sigma_{k}\right)\right)\right]}{\left\langle\sigma_{1} \sigma_{2}\right\rangle \ldots\left\langle\sigma_{k} \sigma_{1}\right\rangle},
$$


where the superfields are integrated along a line in twistor space

$$
\mathcal{Z}\left(\sigma_{i}\right)=\mathcal{Z}_{\alpha} \sigma_{i}^{\alpha}
$$

with $\mathcal{Z}_{\alpha}$ satisfying the incidence relations (2.2) for different $\lambda_{\alpha}$. The measure is $D \sigma=\langle\sigma d \sigma\rangle$ and the bracket notation stands for $\left\langle\sigma_{i} \sigma_{j}\right\rangle=\epsilon_{\alpha \beta} \sigma_{i}^{\alpha} \sigma_{j}^{\beta}$. While non-trivial, it has been shown that any physical quantity is independent of the reference twistor $\mathcal{Z}_{\diamond}$.

The spacetime equations of motion can be obtained from the action above by expanding the superfield $\mathcal{A}$ in components

$$
\mathcal{A}(\mathcal{Z}, \overline{\mathcal{Z}})=a+\chi^{a} \psi_{a}+\frac{1}{2} \chi^{a} \chi^{b} \phi_{a b}+\frac{1}{3 !} \epsilon_{a b c d} \chi^{a} \chi^{b} \chi^{c} \psi^{\prime d}+\frac{1}{4 !} \epsilon_{a b c d} \chi^{a} \chi^{b} \chi^{c} \chi^{d} a^{\prime}
$$

In the formula above the fields on the right-hand side depend on the bosonic twistors $Z, \bar{Z}$ parametrizing a line. Moreover $\left\{a, a^{\prime}\right\}$ are the two gluon helicity states, $\left\{\psi_{a}, \psi^{\prime d}\right\}$ are the gluinos and $\phi_{a b}$ are the six scalars. It is very important to notice that the action above is chiral and it contains the topological term $i F \tilde{F}$, where $F$ is the field strength. While this term is not important in perturbation theory because it is a total derivative, it is going to contribute to the integrand we want to compute by introducing a term proportional to the spacetime tensor $\epsilon^{\mu \nu \rho \lambda}$. These terms have the wrong parity and they integrate to zero [53].

\subsection{Correlation functions of the stress-tensor multiplet}

Our aim is to compute the correlation function of four $\mathbf{2 0}^{\prime}$ operators defined in (1.1). This operator is the lowest component of the stress-tensor supermultiplet $\mathcal{T}$, whose top component is the Lagrangian. The fact that this is a short multiplet implies that $\mathcal{T}$ depend only in half of the odd variables $\theta^{a \alpha}, \bar{\theta}_{a}^{\dot{\alpha}}$.

In order to define the relevant fermionic degrees of freedom, it is convenient to introduce the auxiliary harmonic variables $u_{a}^{b} \equiv\left(u_{a}^{+\mathfrak{b}}, u_{a}^{-\mathfrak{b}^{\prime}}\right)$ which parametrize the coset $\mathrm{SU}(4) /\left(\mathrm{SU}(2) \times \mathrm{SU}(2)^{\prime} \times \mathrm{U}(1)\right)$. The indices $a, b$ are fundamental indices of SU(4), while $\mathfrak{b}, \mathfrak{b}^{\prime}$ are fundamental indices of $\mathrm{SU}(2)$ and $\mathrm{SU}(2)^{\prime}$ respectively, with the signs indicating the $\mathrm{U}(1)$ charge. These variables and their complex conjugates satisfy several unitary and completeness conditions which follow because $u_{a}^{b}$ is in SU(4). The harmonic variables allow us to write manifestly $\mathrm{SU}(4)$ invariant expressions. If one defines

$$
\theta_{\alpha}^{+\mathfrak{b}}=\theta_{\alpha}^{a} u_{a}^{+\mathfrak{b}}, \quad \theta_{\alpha}^{-\mathfrak{b}^{\prime}}=\theta_{\alpha}^{a} u_{a}^{-\mathfrak{b}^{\prime}}
$$

then one can decompose every $\theta_{\alpha}^{a}$ as

$$
\theta_{\alpha}^{a}=\theta_{\alpha}^{+\mathfrak{b}} \bar{u}_{+\mathfrak{b}}^{a}+\theta_{\alpha}^{-\mathfrak{b}^{\prime}} \bar{u}_{-\mathfrak{b}^{\prime}}^{a},
$$

where $\bar{u}$ are the complex conjugates of the harmonic variables $u$. The stress-tensor superfield $\mathcal{T}\left(x, \theta^{+}, \bar{\theta}_{-}, u\right)$ depends on half of the odd variables, both chiral and anti-chiral, but it is useful to focus on the chiral part of the multiplet, where all $\bar{\theta}_{-}$vanish, so that we have

$$
\mathcal{T}\left(x, \theta^{+}, u\right)=\mathcal{O}^{++++}(x)+\ldots+\left(\theta^{+}\right)^{4} \mathcal{L}(x),
$$


and we have omitted the other powers of $\theta^{+}$in the expansion. The field $\mathcal{L}(x)$ is the chiral Lagrangian and $\mathcal{O}^{++++}(x)=\operatorname{Tr}\left(\phi^{++} \phi^{++}\right)$is a representation of the half-BPS operator defined in (1.1) with $\phi^{++}=\phi^{a b} u_{a}^{+\mathfrak{b}} u_{b}^{+\mathfrak{c}} \epsilon_{\mathfrak{b c}}$. The connexion between $u_{b}^{a}$ and $y_{i}$ is made with the following particular parametrisation of the harmonic variables

$$
u_{b}^{+a}=\left(\delta_{\mathfrak{b}}^{\mathfrak{a}}, y_{\mathfrak{b}^{\prime}}^{\mathfrak{a}}\right), \quad u_{b}^{-\mathfrak{a}^{\prime}}=\left(0, \delta_{\mathfrak{b}^{\prime}}^{\mathfrak{a}^{\prime}}\right), \quad \bar{u}_{+\mathfrak{a}}^{b}=\left(\delta_{a}^{b}, 0\right), \quad \bar{u}_{-\mathfrak{a}^{\prime}}^{b}=\left(-y_{\mathfrak{a}^{\prime}}^{\mathfrak{b}}, \delta_{\mathfrak{a}^{\prime}}^{\mathfrak{b}^{\prime}}\right)
$$

with $y^{2}=-y_{\mathfrak{a}^{\mathfrak{b}}}^{\mathfrak{b}} y_{\mathfrak{b}}^{\mathfrak{a}^{\prime}} / 2$ and the indices $\mathfrak{a}, \mathfrak{a}^{\prime}$ are raised and lowered as usual with the epsilon tensors.

The correlation functions $\mathcal{G}_{n}=\langle\mathcal{T}(1) \ldots \mathcal{T}(n)\rangle$ have a series expansion in $\theta_{i}^{+}$as a consequence of (2.11), and we can extract the correlation function of four $\mathbf{2 0}$ operators by computing $\mathcal{G}_{4}$ and reading its lowest component, or equivalently, by sending all $\theta_{i}^{+}$to zero. For four- and higher-point functions there is a dependence on the coupling $a$, which can be made precise through the Lagrangian insertion method

$$
\frac{\partial}{\partial g_{\mathrm{YM}}^{2}} \mathcal{G}_{n}=\int d^{4} x_{n+1}\left\langle\mathcal{T}(1) \ldots \mathcal{T}(n) \mathcal{L}\left(x_{n+1}\right)\right\rangle=\int d^{4} x_{n+1} d^{4} \theta_{n+1}^{+} \mathcal{G}_{n+1},
$$

or, more generally,

$$
\frac{1}{m !} \frac{\partial^{m} \mathcal{G}_{n}}{\partial g_{\mathrm{YM}}^{2 m}}=\int \prod_{i=1}^{m} d^{4} x_{n+i} d^{4} \theta_{n+i}^{+} \mathcal{G}_{n+m} .
$$

On the other hand, from equation (2.5) we can also derive the following insertion formula

$$
\frac{\partial}{\partial g_{\mathrm{YM}}^{2}} \mathcal{G}_{n}=\int d^{4} x_{n+1} d^{8} \theta_{n+1}\left\langle\mathcal{T}(1) \ldots \mathcal{T}(n) L_{\mathrm{int}}\left(x_{n+1}, \theta_{n+1}\right)\right\rangle,
$$

which hints at the following representation of the stress-tensor superfield in twistor space

$$
\mathcal{T}\left(x, \theta^{+}\right)=\int d^{4} \theta^{-} L_{\text {int }}(x, \theta) .
$$

In order to extract the $\ell$-loop four-point function, we will then have to compute the following $(4+\ell)$-point correlator in twistor space

$$
\mathcal{G}_{4+\ell}=\int d^{4} \theta_{1}^{-} \ldots d^{4} \theta_{4+\ell}^{-}\left\langle L_{\text {int }}\left(x_{1}, \theta_{1}\right) \ldots L_{\text {int }}\left(x_{4+\ell}, \theta_{4+\ell}\right)\right\rangle .
$$

Looking at equation (2.5) we see that each $L_{\text {int }}$ comes with at least two superfield insertions along the twistor line. Moreover, according to equation (2.13), we also need to integrate the $\theta^{+}$variables for each of the Lagrangian insertions, which means that at $\ell$ loops we are looking for the component of $\mathcal{G}_{4+\ell}$ with Grassmann degree $4 \ell$. Since each vertex reduces the degree by four units while each propagator increases it by four, then at $\ell$ loops we need to construct all diagrams with $4+\ell$ vertices and $4+2 \ell$ propagators. All such graphs can be generated by the open source Sage [46].

The computation of $\mathcal{G}_{n}$ at tree level is made by summing all relevant diagrams and for each graph in twistor space we have to use the Feynman rules which were derived in [45]:

1. A propagator connecting vertices $i$ and $j$ provides a factor $d_{i j}=y_{i j}^{2} / x_{i j}^{2}$ and a colour delta function $\delta^{a_{i} a_{j}}$, 
2. A bivalent vertex contributes a colour factor $\operatorname{Tr}\left(T^{a_{1}} T^{a_{2}}\right)=\delta^{a_{1} a_{2}}$,

3. Higher-valence vertices are associated with the factor $R_{j_{1} \ldots j_{m}}^{i} \operatorname{Tr}\left(T^{a_{1}} \ldots T^{a_{m}}\right)$, with $T^{a}$ the generators of the gauge group and the $R$ factor defined by

$$
R_{j_{1} j_{2} \cdots j_{k}}^{i}=-\int d^{4} \theta_{i}^{-} \frac{\delta^{2}\left(\left\langle\sigma_{i j_{1}} \theta_{i}^{-}\right\rangle+A_{i j_{1}}\right) \delta^{2}\left(\left\langle\sigma_{i j_{2}} \theta_{i}^{-}\right\rangle+A_{i j_{2}}\right) \ldots \delta^{2}\left(\left\langle\sigma_{i j_{k}} \theta_{i}^{-}\right\rangle+A_{i j_{k}}\right)}{\left\langle\sigma_{i j_{1}} \sigma_{i j_{2}}\right\rangle\left\langle\sigma_{i j_{2}} \sigma_{i j_{3}}\right\rangle \ldots\left\langle\sigma_{i j_{k}} \sigma_{i j_{1}}\right\rangle} .
$$

The delta functions are fermionic and therefore, by construction, $R_{j_{1} j_{2} \cdots j_{k}}^{i}$ has Grassmann degree $2 k-4$. The $\sigma_{i j}$ originate from the integrations in equation (2.6), which are localised by the twistor propagators and become

$$
\sigma_{i j}^{\alpha}=\epsilon^{\alpha \beta} \frac{\left\langle Z_{i, \beta} Z_{\diamond} Z_{j, 1} Z_{j, 2}\right\rangle}{\left\langle Z_{i, 1} Z_{i, 2} Z_{j, 1} Z_{j, 2}\right\rangle},
$$

where $Z_{j, 1}$ and $Z_{j, 2}$ are the bosonic components of the twistors parametrizing a line which corresponds to the spacetime point $x_{j}^{\mu}$. Finally, by setting the fermionic components of the auxiliary supertwistor to zero we have

$$
A_{i j}^{\mathfrak{a}^{\prime}}=\left[\left\langle\sigma_{j i} \theta_{j}^{+\mathfrak{b}}\right\rangle+\left\langle\sigma_{i j} \theta_{i}^{+\mathfrak{b}}\right\rangle\right]\left(y_{i j}^{-1}\right)_{\mathfrak{b}}^{\mathfrak{a}^{\prime}} .
$$

The $R$ factors defined in (2.18) have several important properties and satisfy some identities which can be found in [45]. In what follows we will only need two of these identities. First, since the numerator of (2.18) does not depend on the ordering of $\left\{j_{1}, \ldots, j_{k}\right\}$ then the effect of a permutation $\rho$ on the indices is simply

$$
R_{j_{\rho(1)} \ldots j_{\rho(k)}}^{i}=R_{j_{1} \ldots j_{k}}^{i} \frac{\left\langle\sigma_{i j_{1}} \sigma_{i j_{2}}\right\rangle \ldots\left\langle\sigma_{i j_{k}} \sigma_{i j_{1}}\right\rangle}{\left\langle\sigma_{i j_{\rho(1)}} \sigma_{i j_{\rho(2)}}\right\rangle \ldots\left\langle\sigma_{i j_{\rho(k)}} \sigma_{i j_{\rho(1)}}\right\rangle} .
$$

In this way we can rewrite $R$ factors in a canonical way and reduce the number of fermionic integrations we need to perform. Second, a multi-index $R_{j_{1} j_{2} \cdots j_{k}}^{i}$ can always be factorized as follows

$$
R_{j_{1} j_{2} \cdots j_{k}}^{i}=R_{j_{1} j_{2} j_{3}}^{i} R_{j_{1} j_{3} j_{4}}^{i} \ldots R_{j_{1} j_{k-1} j_{k}}^{i},
$$

which implies that each diagram can be rewritten in terms of a fundamental building block $R_{j_{1} j_{2} j_{3}}^{i}$, which takes the following form after the integration over the $\theta_{i}^{-}$

$$
R_{123}^{i}=-\frac{\delta^{2}\left(\left\langle\sigma_{i 1} \sigma_{i 2}\right\rangle A_{i 3}+\left\langle\sigma_{i 2} \sigma_{i 3}\right\rangle A_{i 1}+\left\langle\sigma_{i 3} \sigma_{i 1}\right\rangle A_{i 2}\right)}{\left\langle\sigma_{i 1} \sigma_{i 2}\right\rangle\left\langle\sigma_{i 2} \sigma_{i 3}\right\rangle\left\langle\sigma_{i 3} \sigma_{i 1}\right\rangle} .
$$

Once we sum all relevant diagrams, we obtain the component of $\mathcal{G}_{4+\ell}$ with fermionic degree $4 \ell$. And since we want to perform the $\int \mathrm{d}^{4} \theta^{+}$integrations at the Lagrangian insertions to obtain the loop-level four-point function, effectively we need to send the $\theta_{i}^{+}$at the external points to zero. This implies that the $A_{i j}^{\mathfrak{a}^{\prime}}$ defined in (2.20) can only give a non-zero contribution if at least one of the indices corresponds to an internal point, which in turn means that we only have dependence on $y_{i j}^{-1}$ if at least one of the indices is from an integrated point. 

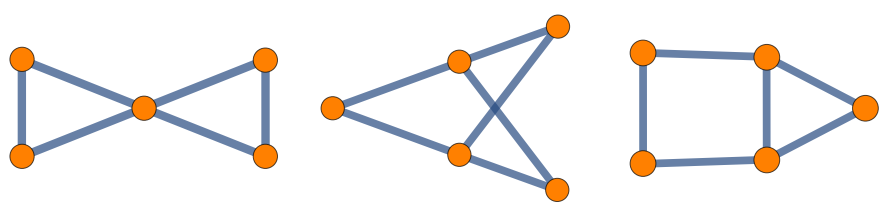

Figure 1. The skeleton graphs used for the one-loop computation of a four-point function. The ribbon graphs are obtained from these by adding colours traces. Only the first graph contributes to the particular choice of polarization, and the only configuration which does not vanish is when the middle vertex corresponds to the integrated Lagrangian insertion. One can easily compute it analytically with equation (B.6).

\begin{tabular}{|ccccc|}
\hline loops & one & two & three & four \\
\hline \# of skeleton graphs & 3 & 11 & 63 & 513 \\
\hline
\end{tabular}

Table 1. The number of skeleton graphs generated with Sage at each loop order. At four loops one can also draw two additional disconnected graphs, however they have to be discarded as they do not contribute to the connected correlator.

Despite the obvious simplicity of this statement, it does imply that if there is a propagator between external points $k$ and $l$, the resulting $y_{k l}^{2}$ factor can never be cancelled as an effect of the fermionic integrations. Consequently, if we choose a particular polarization where $y_{k l}$ vanishes, then we can neglect all diagrams which contain a propagator between those two points. This is a great simplification because the factorized form of the correlator in equation (1.3) allows us to select external polarizations such that only $y_{12}$ and $y_{34}$ are different from zero, thus greatly reducing the number of twistor space diagrams we need to evaluate. In the next subsection, we explain how to compute a four-point function with this assumption. It is possible to perform several intermediate analytical computations as well and we give examples in the appendix B.

\subsection{Four-loop four-point function}

The first step in the calculation of the four-point function is to generate all relevant graphs. As discussed previously, at $\ell$ loops we need graphs with $4+\ell$ vertices and $4+2 \ell$ propagators. These graphs can be easily constructed at lower loops but the number of graphs increases very fast with the loop order, so we used the program Sage [46] to generate them. At one and two loops, the skeleton graphs are shown in figures 1 and 2 respectively, while the number of skeleton graphs up to four loops is shown in table 1. At one loop one can draw graphs with either a single quartic vertex or with two trivalent vertices. The list of skeleton graphs can then be easily generated with Sage in both cases with the code

$$
\begin{aligned}
& \operatorname{graphs}(5, \text { degree_sequence }=[2,2,2,2,4]), \\
& \operatorname{graphs}(5, \text { degree_sequence }=[2,2,2,3,3]) .
\end{aligned}
$$

The next step in the computation is to generate the ribbon graphs from the skeleton graphs obtained with Sage. In other words, each vertex of valence $v$ is assigned both a colour trace $\operatorname{Tr}\left(T^{j_{1}} \ldots T^{j_{v}}\right)$ and an $R_{j_{1} j_{2} \cdots j_{v}}^{i}$ factor as defined in (2.18), and each propagator 


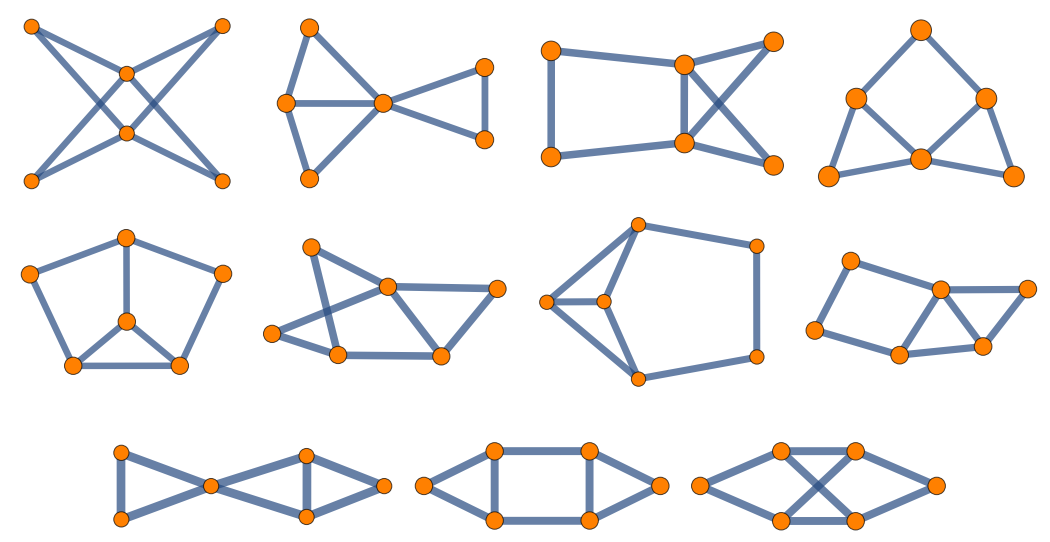

Figure 2. The skeleton graphs at two loops generated by Sage. The final set of ribbon graphs is obtained from these by adding the colour factors and summing over all inequivalent assignments of external and internal points to the vertices.

supplies an additional factor of $d_{i j}$. In general, each skeleton graph obtained with Sage leads to a large number of ribbon graphs. This happens because any inequivalent permutation of the indices $\left\{j_{1}, \ldots, j_{v}\right\}$ appearing in the colour traces and $R$ factors gives rise to a different ribbon graph. More precisely, a skeleton graph with $n$ vertices of valences $\left\{v_{1}, \ldots, v_{n}\right\}$ produces $\prod_{i=1}^{n}\left(v_{i}-1\right)$ ! ribbon graphs, corresponding to the non-cyclic permutations at each vertex.

The graph propagators also provide colour delta functions so that the indices in the colour traces of the vertices are fully contracted. Effectively the color structure of the ribbon graph simplifies to a polynomial in $N_{c}$ through successive application of the fission and fusion rules

$$
\begin{aligned}
\operatorname{Tr}\left(T^{a} B T^{a} C\right) & =\operatorname{Tr}(B) \operatorname{Tr}(C)-\frac{\gamma}{N_{c}} \operatorname{Tr}(B C), \\
\operatorname{Tr}\left(T^{a} B\right) \operatorname{Tr}\left(T^{a} C\right) & =\operatorname{Tr}(B C)-\frac{\gamma}{N_{c}} \operatorname{Tr}(B) \operatorname{Tr}(C),
\end{aligned}
$$

where $B$ and $C$ are arbitrary matrices and the cases $\gamma=0,1$ correspond to $\mathrm{U}\left(N_{c}\right)$ and $\mathrm{SU}\left(N_{c}\right)$ respectively. We have checked that our four-loop four-point correlator is independent of $\gamma$. This seems a bit surprising at the non-planar loop level, but it is true for lenght-two operators. The U(1) part of the gauge group is free and the external operators only give a trivial colour factor $\operatorname{Tr}\left(T^{a_{1}} T^{a_{2}}\right)=\delta^{a_{1} a_{2}}$ in this case. ${ }^{3}$ For correlators involving half-BPS operators of higher weight, we expect that generically the result will depend on $\gamma$, see for example appendix A of [41]. The evaluation of ribbon graphs can be further simplified by using the property of the $R$ factor given in (2.21), which allows us to rewrite all $R$ factors in a chosen canonical order, up to factors of $\left\langle\sigma_{i j} \sigma_{i k}\right\rangle$. This implies that all ribbon graphs which originate from the same skeleton graph will produce the same canonical $R$ factors and differ only by a simple prefactor. In conclusion, to each skeleton graph we associate a canonical ribbon graph. With this knowledge, and without performing any fermionic integration, we could already rederive the well-known result that the correlator of four $\mathbf{2 0}^{\prime}$

\footnotetext{
${ }^{3}$ We thank Sergey Frolov for a discussion on this point.
} 


\begin{tabular}{|ccccc|}
\hline loops & one & two & three & four \\
\hline \# of graphs generic polarization & 45 & 1417 & 75141 & 6019618 \\
\hline \# of graphs particular polarization & 1 & 73 & 7939 & 715350 \\
\hline
\end{tabular}

Table 2. The final number of diagrams which is obtained from canonical ribbon graphs by associating vertices to internal and external positions. In the generic polarization all $y_{i j}$ are non-vanishing, while in the particular polarization any scalar product other than $y_{12}$ and $y_{34}$ is zero. The four-loop correlator in this paper was evaluated in the particular configuration, for which the number of graphs is greatly reduced.

operators can only receive a non-planar correction at four loops. This happens because for lower loop orders all skeleton graphs produce the simple colour factor of $\left(N_{c}^{2}-1\right)$.

Finally, from the canonical ribbon graphs we can generate the final set of diagrams by associating vertices with external and internal positions in all inequivalent ways. Naively this would generate $(4+\ell)$ ! graphs from each canonical ribbon graph contributing to the $\ell$-loop correlator. However, most graphs possess a non-trivial automorphism group, so that the number of inequivalent permutations is effectively much smaller. Moreover, once we have assigned positions to the vertices of the graph we can check if it is possible to produce an $\left(\theta^{+}\right)^{4}$ factor for each of the internal points, and it turns out that this test considerably decreases the number of allowed graphs. The number of final graphs for a generic polarization of the external operators is shown in the first line of table 2. Luckily, as explained in subsection 2.2, we can perform the computation for a particular choice of polarizations and reconstruct the final result unambiguously. If polarizations are such that only $y_{12}$ and $y_{34}$ are non-vanishing, then any graph with external-to-external propagators other than $d_{12}$ or $d_{34}$ is identically zero. This drastically reduces the number of diagrams to compute, as can be seen in table 2 .

After generating all the graphs and their prefactors, the final step in the calculation is to replace the $R$ factors by their expression (2.18) and perform the fermionic integrations in $\theta^{+}$at the Lagrangian insertions. At four loops, all graphs become a product of eight $R_{j_{1} j_{2} j_{3}}^{i}$ factors, thus producing many terms with the correct number of $\theta_{i}^{+}$. An analytical computation is then very hard, especially because the dependence on the auxiliary twistor $\mathcal{Z}_{\diamond}$ only disappears after summing many graphs, see [45] for details. Therefore, in this work we have performed the computation numerically by giving integer values to all components of the polarization and position vectors. While it is not necessary to restrict to integer numbers, we found that was a practical way of avoiding numerical fluctuations and errors.

The planar integrand had already been fixed in [4], and we successfully reproduced their result up to four loops with our diagrammatic expansion in twistor space. This important comparison provides a good cross-check of our implementation. At the nonplanar level the integrand was written as a linear combination of four polynomials with undetermined coefficients. By computing each twistor diagram for twelve different sets of numerical values we produced an overcomplete system of equations and were able to fix those coefficients. Each numerical evaluation of the graphs took approximately 3 days in a single computer with 20 cores. The result is given in the following section, where we cross-check it further against some available non-planar data. 
If we consider configurations of points living in four dimensions, then there is a technical issue that arises. As discussed at the end of subsection 2.1, the twistor action has the topological term $i F \tilde{F}$ and so the evaluation of the graphs generates terms involving the tensor $\epsilon^{\mu \nu \rho \lambda}$. These are spurious contributions at the level of the integrand and they must be absent in the final integrated result, i.e. they necessarily lead to functions that integrate to zero. In other words, the Lagrangians with and without the term $i F \tilde{F}$ are both equally valid objects and produce the same perturbative expansion since $i F \tilde{F}=\partial_{\mu} K^{\mu}$ for some $K^{\mu}$, and such a term cannot give any non-trivial perturbative contribution as it is a total derivative over the integration point in the Lagrangian insertion method (this term can however be important nonperturbatively $[54,55]$ ). Note that in Lorentzian signature the terms with an odd number of epsilon tensors will be imaginary, which means that we can single out their contribution in our calculations. In order to fix them we found the complete basis of pseudoscalar conformal integrands with extended permutation symmetry. At first there seem to be many structures one can form, but when we implement permutation symmetry we must include minus signs to compensate the antisymmetry of the epsilon tensor, and at the end there are only 4 such polynomials. Furthermore, two of those correspond to pseudoscalar conformal Gram polynomials, leaving only two degrees of freedom. We obtained an imaginary non-planar component for five of our numerical data points, thus producing an overcomplete system of equations. Effectively, the result is a shift to the polynomial $P^{(1,4)}$ by

$$
\begin{aligned}
\tilde{P}^{(1,4)}\left(x_{i}\right)= & \frac{12 i}{14}\left(x_{17}^{2} x_{18}^{2} x_{26}^{2} x_{28}^{2} x_{35}^{2} x_{37}^{2} x_{45}^{2} x_{46}^{2} x_{78}^{2} \epsilon_{123456}^{c}+S_{8} \text { perms }\right) \\
& +\frac{18 i}{14}\left(x_{18}^{4} x_{27}^{2} x_{28}^{2} x_{36}^{2} x_{37}^{2} x_{45}^{2} x_{47}^{2} x_{56}^{2} \epsilon_{123456}^{c}+S_{8} \text { perms }\right) .
\end{aligned}
$$

where we defined an $\epsilon^{c}$ tensor by using six-dimensional embedding vectors $\left(1, x^{2}, x^{\mu}\right)$

$$
\epsilon_{i j k l m n}^{c}=\left|\begin{array}{cccc}
1 & 1 & \cdots & 1 \\
x_{i}^{2} & x_{j}^{2} & \cdots & x_{n}^{2} \\
x_{i}^{\mu} & x_{j}^{\mu} & \cdots & x_{n}^{\mu}
\end{array}\right| .
$$

By constructing the pseudoscalar polynomials with this six-dimensional epsilon tensor we guarantee that the polynomial in (2.26) is conformal. In addition, recall that a product of ordinary epsilon tensors can always be reduced to a sum of products of Kronecker deltas

$$
\epsilon_{i_{1} \ldots i_{n}} \epsilon^{j_{1} \ldots j_{n}}=n ! \delta_{\left[i_{1}\right.}^{j_{1}} \cdots \delta_{\left.i_{n}\right]}^{j_{n}} .
$$

Thus, if spurious terms are generate in this way, the conformal and permutation symmetries ensure they can be written as a linear combination of the 32 four-loop polynomials from [4]. Since our method relies on a numerical evaluation and subsequent fitting against a basis of polynomials, it is crucial to make sure that those spurious terms do not affect our result. We have analysed the 32 polynomials and apart from the conformal Gram polynomials, there is at most one linear combination that could integrate to zero. Thus we are confident that our twelve data points still give an overcomplete set of equations, even when we consider an extended basis of polynomials which includes the term that integrates to zero. 
Finally, we performed a few additional numerical tests by checking invariance under a change of polarizations for the internal vertices and independence of the result on the auxiliary twistor.

\section{OPE analysis}

The diagrammatic computation of the previous section fixed the non-planar polynomial to be

$$
P^{(1,4)}\left(x_{i}\right)=-6 Q_{4}\left(x_{i}\right)
$$

with $Q_{4}\left(x_{i}\right)$ defined in (1.8). However, some of the terms in the polynomial lead to pseudoconformal integrals. The weight at each integrated point is +4 , but those integrals are divergent in four dimensions and once we introduce dimensional regularization they lose their conformal properties. Since it is more cumbersome to deal with pseudo-conformal integrals, we chose to add a conformal Gram polynomial to the integrand and rewrite it as

$$
P^{(1,4)}\left(x_{i}\right)=2 \sum_{j=1}^{32} \tilde{q}_{j} P_{j}^{(4)}\left(x_{i}\right),
$$

with $P_{j}^{(4)}$ defined in equations (5.9) and (C.1) of [4] and the coefficients $\tilde{q}_{j}$ given by

$$
\tilde{q}=\left\{6,6,-6,8,0,6,0,-1,-2,0^{2}, 2,-1,0^{4}, 2,2,-2,-4,0,-2,0^{3},-48,-4,0,4,0,0\right\} .
$$

While the Gram polynomials vanish in four dimensions, the presence of pseudo-conformal integrals and dimensional regularization means that at the finite order they could in principle be non-vanishing. However, we expanded all integrals to the third order in spin and observed that, up to that order, the finite piece is indeed zero. The non-trivial character of the cancellation gives us confidence that it will persist at any order in spin, thus allowing our rewriting of equation (3.2). The conformal Gram polynomials parametrize a three-dimensional subspace of the allowed polynomials, and we used two of its degrees of freedom to ensure that none of the pseudo-conformal integrals contributes in (3.3). The last parameter is chosen in a way that eliminates some of the more difficult conformal integrals.

While the twistor space method allowed us to obtain the integrand of the correlation function, we are still faced with the integration of the Lagrangian insertions. Conformal symmetry reduces the complexity of the problem by restricting to functions of two crossratios only

$$
u=\frac{x_{12}^{2} x_{34}^{2}}{x_{13}^{2} x_{24}^{2}}, \quad v=\frac{x_{14}^{2} x_{23}^{2}}{x_{13}^{2} x_{24}^{2}}
$$

The four-loop ladder diagram is known exactly [56], and recently the method of differential equations was used to fix a different topology [57], but in general the evaluation of four-loop four-point integrals is a daunting task.

Therefore, in this work we will focus on the Euclidean coincidence limit, which is tractable. We use the freedom of conformal symmetry to send $x_{4}$ to infinity, so that 
effectively we deal only with three-point integrals, and then we let $x_{1}$ approach $x_{2}$. In that case the cross-ratios become

$$
u=\frac{x_{12}^{2}}{x_{13}^{2}} \rightarrow 0, \quad v=1-Y=\frac{x_{23}^{2}}{x_{13}^{2}} \rightarrow 1 .
$$

Each of the conformal integrals has now two distinct scales $\left|x_{12}\right| \ll\left|x_{13}\right|$, which means that we can approximate the integrals with the method of asymptotic expansions. The idea is that for each integration variable $x_{i}$ we can now divide the integration domain into two regions, one where $x_{1 i}$ is of the order of $x_{12}$, and another where it is of the order of $x_{13}$. In that way, an $\ell$-loop conformal integral will split into $2^{\ell}$ terms, corresponding to different distributions of the integration variables in the two regions. For example, if $x_{1 i}$ is of the order of $x_{12}$ and $x_{1 j}$ of the order of $x_{13}$, we can Taylor expand the propagator

$$
\frac{1}{x_{i j}^{2}}=\frac{1}{x_{1 j}^{2}} \sum_{n=0}^{\infty}\left(\frac{2 x_{1 i} \cdot x_{1 j}-x_{1 i}^{2}}{x_{1 j}^{2}}\right)^{n} .
$$

This Taylor expansion is only convergent for $\left|x_{1 i}\right| \leq\left|x_{1 j}\right|$, but we extend the integration domain in this region to the whole space. That makes the integrals strictly divergent, which can be resolved by introducing dimensional regularization and requiring that scaleless integrals vanish [58]. In order to find the lowest order in the small $u$ expansion we can ignore the $x_{1 i}^{2}$ terms from the numerator of (3.6), and higher powers in the Taylor expansion will contribute to higher orders in the small $Y$ expansion.

Looking at equation (3.6) we realize that the two regions are effectively disentangled. This indicates that a term with a $\{k, \ell-k\}$ split of the integration variables into the $\left\{x_{12}, x_{13}\right\}$ regions will lead to a product of $k$ - and $(\ell-k)$-loop two-point integrals, with external points $x_{1}$ and $x_{2}$, or $x_{1}$ and $x_{3}$, respectively. This is a considerable simplification because we are able to approximate the conformal integral with single-scale integrals. The two-point integrals will generically have numerators with tensor structures, but we can follow the strategy of [21] to reduce them to scalar integrals. Once that is accomplished we use LiteRed [59] and FIRE [60] to implement IBP identities and reduce to a basis of simpler master integrals, which were obtained in [61].

For simplicity we focused on a parametrization of the integrand which involved solely convergent integrals, but that was not strictly necessary. The method of asymptotic expansions can also be applied to pseudo-conformal integrals and the only caveat is that one must disregard conformal symmetry. More specifically, different terms in the integrand lead to integrals which are the same up to a permutation of the external points. When they are convergent one can rely on the invariance of the cross-ratios under the group of double-transpositions

$$
\{\mathrm{id},(12)(34),(13)(24),(14)(23)\}
$$

to relate them. However, the pseudo-integrals are divergent and in dimensional regularization they are not simply a function of cross ratios, which means that we must perform the asymptotic expansion independently for each permutation of the external points. Furthermore, the expansions of some pseudo-conformal integrals start at $u^{-1}$, and while this 
spurious pole cancels in the Gram and non-planar polynomials, we do need to expand those integrals at a higher order in $u$ in order to find their contribution at leading twist. We expanded all integrals to order $u^{0} Y^{2}$ but, after confirming that the Gram polynomials still vanish in dimensional regularization, we chose to perform the expansions to order $u^{0} Y^{6}$ on a suitable parametrization of the integrand which avoids these complications.

Since $\mathcal{N}=4 \mathrm{SYM}$ is a conformal theory, we can use the OPE to rewrite the four-point function as

$$
G_{4}=\frac{y_{12}^{4} y_{34}^{4}}{x_{12}^{4} x_{34}^{4}} \sum_{\Delta, l, n, m} C_{\mathcal{O}_{20^{\prime}} \mathcal{O}_{20^{\prime}} \mathcal{O}}^{2} g_{\Delta, l}(u, v) Y_{n, m}(\sigma, \tau),
$$

where $\sigma$ and $\tau$ are the $R$-symmetry cross-ratios, $Y_{n, m}$ is the $R$-symmetry block for the exchange of an operator in the $\mathrm{SU}(4)$ representation $[n-m, 2 m, n-m]$, and $g_{\Delta, l}$ is the conformal block for an operator of dimension $\Delta$ and spin $l$. In the Euclidean OPE limit that was described above the conformal block simplifies to

$$
g_{\Delta, l}(u, Y) \approx u^{\frac{\Delta-l}{2}} Y_{2}^{l} F_{1}\left(\frac{\Delta+S}{2}, \frac{\Delta+S}{2} ; \Delta+S ; Y\right),
$$

which means that only the lowest-twist non-protected operators contribute to the leading $u$ behaviour of the four-loop correlator. Furthermore, a suitable choice of the external polarization vectors $y_{i}$ allows us to single out the $[0,2,0]$ representation. Since there is a single twist-two operator in the $20^{\prime}$ representation for each spin, we are able to extract all their OPE coefficients and anomalous dimensions.

The OPE data is written as a double expansion on the genus and coupling constant

$$
\begin{aligned}
\Delta(l) & =\Delta_{l}^{(0)}+\sum_{g=0}^{\infty} \sum_{\ell=1}^{\infty} \frac{a^{\ell}}{N_{c}^{2 g}} \gamma_{l}^{(g, \ell)}, \\
C_{\mathcal{O}_{20^{\prime}} \mathcal{O}_{20^{\prime}} \mathcal{O}_{\Delta, l}}^{2} & =\sum_{g=0}^{\infty} \sum_{\ell=0}^{\infty} \frac{a^{\ell}}{N_{c}^{2 g}} \alpha_{l}^{(g, \ell)} .
\end{aligned}
$$

Note that with the twistor space calculation we reconstruct the full $N_{c}$ dependence of the four-loop four-point function, which shows that the genus expansion truncates at the first non-planar order. The non-planar anomalous dimensions are therefore

$$
\begin{aligned}
\gamma_{2}^{(1,4)} & =-17280 \zeta_{5}, \\
\gamma_{4}^{(1,4)} & =2800+\frac{28000 \zeta_{3}}{3}-\frac{100000 \zeta_{5}}{3}, \\
\gamma_{6}^{(1,4)} & =\frac{132986}{25}+\frac{85064 \zeta_{3}}{5}-\frac{230496 \zeta_{5}}{5}, \\
\gamma_{8}^{(1,4)} & =\frac{220854227}{29400}+\frac{164142 \zeta_{3}}{7}-\frac{13898904 \zeta_{5}}{245} .
\end{aligned}
$$

These results match the perturbative computation of Velizhanin [28-30] for spin 2, 4 and $6{ }^{4}$ Meanwhile the expression for spin 8 is new and its leading transcendental piece matches Velizhanin's conjecture for general spin

$$
\left.\gamma_{l}^{(1,4)}\right|_{\zeta_{5}}=-7680 S_{1}(l)^{2}
$$

\footnotetext{
${ }^{4}$ See footnote 1 .
} 
with $S_{1}(l)$ the harmonic sum. This seems to imply the behaviour $\log ^{2}(l)$ for large spin, which is in contradiction wih the expected one. However, this is only part of the result and cancellations can occur at large spin. We also extracted the non-planar correction to the OPE coefficients, all of which are novel results,

$$
\begin{aligned}
& \alpha_{2}^{(1,4)}=5760 \zeta_{5}+5040 \zeta_{7}, \\
& \alpha_{4}^{(1,4)}=\frac{112}{3}+\frac{400 \zeta_{3}}{9}+\frac{205040 \zeta_{5}}{441}+600 \zeta_{7}, \\
& \alpha_{6}^{(1,4)}=\frac{48821149}{6534000}+\frac{41643 \zeta_{3}}{3025}+\frac{191044 \zeta_{5}}{9075}+\frac{588 \zeta_{7}}{11}, \\
& \alpha_{8}^{(1,4)}=\frac{25811374441}{28171962000}+\frac{35869013 \zeta_{3}}{18632250}+\frac{477038734 \zeta_{5}}{2630252625}+\frac{3044 \zeta_{7}}{715} .
\end{aligned}
$$

The transcendental structure of the spin 2 structure constant is quite interesting due to the absence of rational and $\zeta_{3}$ terms, but we do not currently understand why that happens. It is possible to derive a closed expression for the $\zeta_{7}$ part of the OPE coefficient, which is given by ${ }^{5}$

$$
\left.\alpha_{l}^{(1,4)}\right|_{\zeta_{7}}=10080 \alpha_{l}^{(0,0)} S_{1}(l)
$$

with the tree-level planar coefficient given by $\alpha_{l}^{(0,0)}=2(l !)^{2} /(2 l) !$.

Finally, we were able to perform the asymptotic expansion of the integrand up to spin 12 , but the IBP reduction of the resulting two-point integrals was not possible. The data we have available is not sufficient to reconstruct an expression for generic spin in terms of harmonic sums, which means that we cannot access the large spin limit of the anomalous dimensions. Perhaps the method of differential equations can be used to find a higher-order expansion of the conformal integrals in a more efficient way.

\section{Conclusion}

In this work, we have fixed the four-loop non-planar integrand of the four-point function of length-two half-BPS operators by a direct computation in twistor space. We performed the Grassmann integrations for numeric vales of the position and polarization vectors and fit the results obtained against a polynomial ansatz with four unknown coefficients. In this formalism each individual graph preserves $\mathcal{N}=4$ superconformal symmetry, apart from the reference twistor. The calculation was done with a particular choice of external polarizations in order to reduce the number of graphs, but the general result can be unambiguously reconstructed due to the factorized dependence on the polarizations in equation (1.4). In principle, one can also use our method to compute higher-point and higher-loop integrands, both at the planar and non-planar level. However, the number of diagrams can grow a lot in those cases and the complexity of the fermionic integrations can also increase considerably. In our case, the integration took an average of three days on 20 cores for each set of numerical values. In order to compute more complicated correlators it is perhaps necessary to implement a method which mixes numerical and analytical methods. More specifically,

\footnotetext{
${ }^{5}$ The expression was found by Gregory Korchemsky and we thank him for communicating it to us.
} 
one can first perform some of the fermionic integrations analytically, as in the examples of appendix B, and then complete the calculation numerically.

Let us now stress a technical detail of our method that could also show up for other correlators. The twistor action from equation (2.5) is chiral and contains the topological term $i F \tilde{F}$ when expanded in components. This implies that terms with $\epsilon^{\mu \nu \rho \lambda}$ can be generated in twistor space calculations. In this work we were able to write down an ansatz for these terms and found the contribution given in (2.26). This terms must integrate to zero and they do not appear in the final integrand, but in principle it can be difficult to isolate the epsilon terms from numerical calculations. However, when working in Lorentzian signature, the terms with an odd number of epsilon tensors have an imaginary contribution and they can be easily isolated. On the other hand, terms with an even number of epsilons give a real contribution which can be rewritten in the usual polynomial basis. Notice that these terms are identically zero when we restrict to a three-dimensial subspace, which implies that this possible spurious contribution can be written in terms of three-dimensional conformal Gram polynomials. A careful analysis using an extended basis showed that our result is not contaminated by this type of terms.

In this work we focused on the non-planar corrections to the four-point function of $20^{\prime}$ operators, which start at four loops. There are also results in the literature for correlation functions of length- $k$ half-BPS operators and for generic $k$ the non-trivial non-planar corrections usually start at lower loops. At the planar level, all four-point functions of such operators are know up to five loops $[62,63]$ while at the non-planar level correlators of four length- $k$ operators are known up to two loops only [64, 65]. It would be very interesting to compute non-planar corrections to more general four-point functions of half-BPS operators at two and higher loops. In that way we would further test the integrability approach to non-planar correlators developed in [40,41], and it could also help understand why nonplanar corrections show up at specific loop orders in this approach. Maybe some of these computations can be done using the twistor reformulation of $\mathcal{N}=4$ SYM used in this paper together wih the prescription for composite operators in twistor space of [66-68].

Finally, it would be extremely nice to obtain a closed-form expression for the nonplanar anomalous dimension and structure constants of twist operators. This would allow to extract the non-planar cusp anomalous dimension and it would give valuable data for an integrability approach to the non-planar spectrum. We have made an OPE analysis of the four-point function and we obtained a few data points. However, that data was not sufficient to completely fix the anomalous dimension at generic spin. It seems hard to push the OPE expansion further with the method of asymptotic expansions and so a new strategy is very likely needed. We hope to return to this point in the future.

\section{Acknowledgments}

We thank Andrei Belitsky, Sergey Frolov, Paul Heslop and Gregory Korchemsky for useful discussions and especially acknowledge Vasco Gonçalves for suggesting the project and for participating at an early stage. We also thank Vasco Gonçalves and Gregory Korchemsky for valuable comments on the manuscript. T.F would like to thank the warm hospitality 
of the Arizona State University where this work was finished. This work was supported by the Serrapilheira Institute (grant number Serra-1812-26900). R.P. is supported by SFI grant $15 / \mathrm{CDA} / 3472$.

\section{A Conventions}

In this work, we have used the following conventions to raise and lower $\mathrm{SU}(2)$ indices

$$
x_{\alpha \dot{\alpha}}=x_{\mu} \sigma_{\alpha \dot{\alpha}}^{\mu}, \quad x^{\dot{\alpha} \alpha}=\epsilon^{\alpha \beta} x_{\beta \dot{\beta}} \epsilon^{\dot{\beta} \dot{\alpha}}, \quad x_{\alpha \dot{\alpha}}=\epsilon_{\dot{\alpha} \dot{\beta}} x^{\dot{\beta} \beta} \epsilon_{\beta \alpha} .
$$

Similarly, for the $y_{\mathfrak{b}^{\prime}}^{\mathfrak{a}}$ variables introduced in (2.12) as a parametrization of the harmonic variables we have

$$
y_{\mathfrak{b}}^{\mathfrak{a}^{\prime}}=y_{\mathfrak{b}^{\prime}}^{\mathfrak{a}} \epsilon^{\mathfrak{G}^{\prime} \mathfrak{a}^{\prime}} \epsilon_{\mathfrak{a} \mathfrak{b}}, \quad y^{2}=-y_{\mathfrak{a}^{\prime}}^{\mathfrak{b}} y_{\mathfrak{b}}^{\mathfrak{a}^{\prime}} / 2 .
$$

The epsilon tensors are defined with $\epsilon_{12}=\epsilon^{12}=1$, so that they obey

$$
\epsilon_{a b} \epsilon^{a c}=\delta_{b}^{c}
$$

For both the spacetime and $R$-symmetry matrices we use the following short-hand notation

$$
x_{i j}^{\dot{\alpha} \alpha}=x_{i}^{\dot{\alpha} \alpha}-x_{j}^{\dot{\alpha} \alpha}, \quad \text { and } \quad\left(y_{i j}\right)_{\mathfrak{b}^{\prime}}^{\mathfrak{a}}=\left(y_{i}\right)_{\mathfrak{b}^{\prime}}^{\mathfrak{a}}-\left(y_{j}\right)_{\mathfrak{b}^{\prime}}^{\mathfrak{a}} .
$$

Using the properties of the Pauli matrices it is possible to show that

$$
x_{\alpha}^{\dot{\alpha}} y_{\dot{\alpha}}^{\alpha}=-2 x \cdot y, \quad x^{\dot{\alpha} \alpha} x_{\alpha}^{\dot{\beta}}=x^{2} \epsilon^{\dot{\alpha} \dot{\beta}},
$$

We can manipulate the last equation above to see that

$$
x^{\dot{\alpha} \alpha}\left(\frac{1}{x^{2}} x_{\alpha \dot{\gamma}}\right)=\delta_{\dot{\gamma}}^{\dot{\alpha}}, \quad \rightarrow \quad\left(x^{-1}\right)_{\alpha \dot{\alpha}}=\frac{1}{x^{2}} x_{\alpha \dot{\alpha}} .
$$

Analogously, one has

$$
\left(y_{i j}^{-1}\right)_{\mathfrak{b}}^{\mathfrak{a}^{\prime}}=\frac{1}{y_{i j}^{2}}\left(y_{i j}\right)_{\mathfrak{b}}^{\mathfrak{a}^{\prime}}
$$

Concerning the integrations of the Grassmann variables $\theta_{i, \alpha}^{ \pm a}$, we use the convention

$$
\int \mathrm{d}^{4} \theta_{i}^{ \pm}=\int \mathrm{d} \theta_{i, 1}^{ \pm 1} \mathrm{~d} \theta_{i, 1}^{ \pm 2} \mathrm{~d} \theta_{i, 2}^{ \pm 1} \mathrm{~d} \theta_{i, 2}^{ \pm 2}
$$

This implies that $\theta_{1}^{ \pm 1} \theta_{1}^{ \pm 2} \theta_{2}^{ \pm 1} \theta_{2}^{ \pm 2}$ integrates to one.

\section{B Examples of fermionic integrations}

In this work we have computed all necessary diagrams numerically, i.e we have set all components of the position and polarization vectors to integer values. Nevertheless, for a given diagram it might be possible to perform some or even all the fermionic integrations analytically. Eventually one can combine the two methods in an efficient way and reduce the complexity of many graphs. Here, we give some examples of integrations that can be 
performed easily. First, consider the $\theta_{i}^{+}$integration when the internal point $i$ is a bivalent vertex. In that case the Grassmann variables can be found in the product of $R$ factors from the adjacent vertices $j$ and $k$

$$
R_{a_{1} \ldots a_{m} i}^{j} R_{b_{1} \ldots b_{n} i}^{k}
$$

From equation $(2.18)$ we can see that the relevant terms for the integration of $\theta_{i}^{+}$come solely from

$$
\delta^{2}\left(\left\langle\sigma_{k i} \theta_{k}^{-}\right\rangle+A_{k i}\right) \delta^{2}\left(\left\langle\sigma_{j i} \theta_{j}^{-}\right\rangle+A_{j i}\right),
$$

which means that the only term with four $\theta_{i}^{+}$is

$$
\left\langle\sigma_{i k} \theta_{i}^{+\mathfrak{a}}\right\rangle\left(y_{k i}^{-1}\right)_{\mathfrak{a}}^{1^{\prime}}\left\langle\sigma_{i k} \theta_{i}^{+\mathfrak{b}}\right\rangle\left(y_{k i}^{-1}\right)_{\mathfrak{b}}^{2^{\prime}}\left\langle\sigma_{i j} \theta_{i}^{+\mathfrak{c}}\right\rangle\left(y_{j i}^{-1}\right)_{\mathfrak{c}}^{1^{\prime}}\left\langle\sigma_{i j} \theta_{i}^{+\mathfrak{d}}\right\rangle\left(y_{j i}^{-1}\right)_{\mathfrak{d}}^{2^{\prime}}=\left(\theta_{i}^{+}\right)^{4} \frac{\left\langle\sigma_{i k} \sigma_{i j}\right\rangle^{2}}{y_{k i}^{2} y_{j i}^{2}}
$$

Therefore, the fermionic integration gives

$$
\int \mathrm{d}^{4} \theta_{i}^{+} R_{a_{1} \ldots a_{m} i}^{j} R_{b_{1} \ldots b_{n} i}^{k}=R_{a_{1} \ldots a_{m}}^{j} R_{b_{1} \ldots b_{n}}^{k} \frac{\left\langle\sigma_{i k} \sigma_{i j}\right\rangle^{2}}{y_{k i}^{2} y_{j i}^{2}} \frac{\left\langle\sigma_{j a_{m}} \sigma_{j a_{1}}\right\rangle}{\left\langle\sigma_{j a_{m}} \sigma_{j i}\right\rangle\left\langle\sigma_{j i} \sigma_{j a_{1}}\right\rangle} \frac{\left\langle\sigma_{k b_{n}} \sigma_{k b_{1}}\right\rangle}{\left\langle\sigma_{k b_{n}} \sigma_{k i}\right\rangle\left\langle\sigma_{k i} \sigma_{k b_{1}}\right\rangle} .
$$

Another example of a simple $\theta_{i}^{+}$integration consists of the internal point sitting at a trivalent vertex connected to two bivalent vertices $j$ and $l$ and one higher-valence denoted by $k$. In that case the necessary fermionic variables are provided by the product of two $R$ factors which integrates to

$$
\int d^{4} \theta_{i}^{+} R_{j k l}^{i} R_{a_{1} \ldots a_{n} i}^{k}=R_{a_{1} \ldots a_{n}}^{k} \frac{y_{j l}^{2}\left\langle\sigma_{i j} \sigma_{i k}\right\rangle\left\langle\sigma_{i k} \sigma_{i l}\right\rangle}{y_{i j}^{2} y_{i k}^{2} y_{i l}^{2}\left\langle\sigma_{i j} \sigma_{i l}\right\rangle} \frac{\left\langle\sigma_{k a_{n}} \sigma_{k a_{1}}\right\rangle}{\left\langle\sigma_{k a_{n}} \sigma_{k i}\right\rangle\left\langle\sigma_{k i} \sigma_{k a_{1}}\right\rangle} .
$$

As the final example, let us now consider the $\theta_{i}^{+}$integration when the internal point $i$ is a quartic vertex connected to bivalent vertices only. In that case the Grassmann variables originate from the $R$ factor at the internal vertex $i$ and we have

$$
\int \mathrm{d}^{4} \theta_{i}^{+} R_{1234}^{i}=\frac{1}{y_{i 1}^{2} y_{i 2}^{2} y_{i 3}^{2} y_{i 4}^{2}}\left(y_{12}^{2} y_{34}^{2} \frac{\left\langle\sigma_{i 1} \sigma_{i 3}\right\rangle\left\langle\sigma_{i 2} \sigma_{i 4}\right\rangle}{\left\langle\sigma_{i 1} \sigma_{i 2}\right\rangle\left\langle\sigma_{i 3} \sigma_{i 4}\right\rangle}+y_{13}^{2} y_{24}^{2}+y_{14}^{2} y_{23}^{2} \frac{\left\langle\sigma_{i 1} \sigma_{i 3}\right\rangle\left\langle\sigma_{i 2} \sigma_{i 4}\right\rangle}{\left\langle\sigma_{i 1} \sigma_{i 4}\right\rangle\left\langle\sigma_{i 2} \sigma_{i 3}\right\rangle}\right) \text {. }
$$

Open Access. This article is distributed under the terms of the Creative Commons Attribution License (CC-BY 4.0), which permits any use, distribution and reproduction in any medium, provided the original author(s) and source are credited.

\section{References}

[1] F. Gonzalez-Rey, I.Y. Park and K. Schalm, A note on four point functions of conformal operators in $N=4$ super Yang-Mills, Phys. Lett. B 448 (1999) 37 [hep-th/9811155] [INSPIRE].

[2] B. Eden, C. Schubert and E. Sokatchev, Three loop four point correlator in $N=4 S Y M$, Phys. Lett. B 482 (2000) 309 [hep-th/0003096] [INSPIRE]. 
[3] B. Eden, P. Heslop, G.P. Korchemsky and E. Sokatchev, Hidden symmetry of four-point correlation functions and amplitudes in $N=4$ SYM, Nucl. Phys. B 862 (2012) 193 [arXiv:1108.3557] [INSPIRE].

[4] B. Eden, P. Heslop, G.P. Korchemsky and E. Sokatchev, Constructing the correlation function of four stress-tensor multiplets and the four-particle amplitude in $N=4$ SYM, Nucl. Phys. B 862 (2012) 450 [arXiv:1201.5329] [INSPIRE].

[5] J.L. Bourjaily, P. Heslop and V.-V. Tran, Amplitudes and correlators to ten loops using simple, graphical bootstraps, JHEP 11 (2016) 125 [arXiv: 1609.00007] [INSPIRE].

[6] D.Z. Freedman, S.D. Mathur, A. Matusis and L. Rastelli, Correlation functions in the $C F T_{d} / A d S_{d+1}$ correspondence, Nucl. Phys. B 546 (1999) 96 [hep-th/9804058] [INSPIRE].

[7] S. Lee, S. Minwalla, M. Rangamani and N. Seiberg, Three point functions of chiral operators in $D=4, N=4 S Y M$ at large $N$, Adv. Theor. Math. Phys. 2 (1998) 697 [hep-th/9806074] [INSPIRE].

[8] G. Arutyunov and S. Frolov, Scalar quartic couplings in type IIB supergravity on $A d S_{5} \times S^{5}$, Nucl. Phys. B 579 (2000) 117 [hep-th/9912210] [InSPIRE].

[9] E. D'Hoker, D.Z. Freedman, S.D. Mathur, A. Matusis and L. Rastelli, Graviton exchange and complete four point functions in the AdS/CFT correspondence, Nucl. Phys. B 562 (1999) 353 [hep-th/9903196] [INSPIRE].

[10] G. Arutyunov and S. Frolov, Four point functions of lowest weight CPOs in $N=4 S Y M(4)$ in supergravity approximation, Phys. Rev. D 62 (2000) 064016 [hep-th/0002170] [INSPIRE].

[11] V. Gonçalves, Four point function of $N=4$ stress-tensor multiplet at strong coupling, JHEP 04 (2015) 150 [arXiv:1411.1675] [inSPIRE].

[12] L. Rastelli and X. Zhou, Mellin amplitudes for $A d S_{5} \times S^{5}$, Phys. Rev. Lett. 118 (2017) 091602 [arXiv: 1608.06624] [INSPIRE].

[13] F. Aprile, J.M. Drummond, P. Heslop and H. Paul, Quantum gravity from conformal field theory, JHEP 01 (2018) 035 [arXiv:1706.02822] [INSPIRE].

[14] L.F. Alday and A. Bissi, Loop corrections to supergravity on $A d S_{5} \times S^{5}$, Phys. Rev. Lett. 119 (2017) 171601 [arXiv: 1706. 02388] [INSPIRE].

[15] L.F. Alday and S. Caron-Huot, Gravitational S-matrix from CFT dispersion relations, JHEP 12 (2018) 017 [arXiv: 1711.02031] [INSPIRE].

[16] L.F. Alday, On genus-one string amplitudes on $A d S_{5} \times S^{5}$, arXiv:1812.11783 [INSPIRE].

[17] D.J. Binder, S.M. Chester, S.S. Pufu and Y. Wang, $N=4$ super-Yang-Mills correlators at strong coupling from string theory and localization, JHEP 12 (2019) 119 [arXiv: 1902.06263] [INSPIRE].

[18] V. Gonçalves, R. Pereira and X. Zhou, $20^{\prime}$ five-point function from $A d S_{5} \times S^{5}$ supergravity, JHEP 10 (2019) 247 [arXiv:1906.05305] [INSPIRE].

[19] S.M. Chester, Genus-2 holographic correlator on $A d S_{5} \times S^{5}$ from localization, arXiv: 1908.05247 [INSPIRE].

[20] M. Baggio, J. de Boer and K. Papadodimas, A non-renormalization theorem for chiral primary 3-point functions, JHEP 07 (2012) 137 [arXiv:1203.1036] [INSPIRE].

[21] B. Eden, Three-loop universal structure constants in $N=4$ SUSY Yang-Mills theory, arXiv: 1207.3112 [INSPIRE]. 
[22] V. Gonçalves, Extracting OPE coefficient of Konishi at four loops, JHEP 03 (2017) 079 [arXiv: 1607.02195] [INSPIRE].

[23] B. Eden and F. Paul, Half-BPS half-BPS twist two at four loops in $N=4 S Y M$, arXiv: 1608.04222 [INSPIRE].

[24] A. Georgoudis, V. Goncalves and R. Pereira, Konishi OPE coefficient at the five loop order, JHEP 11 (2018) 184 [arXiv:1710.06419] [INSPIRE].

[25] M.S. Bianchi, A note on three-point functions of unprotected operators, JHEP 03 (2019) 154 [arXiv: 1809.04376] [INSPIRE].

[26] M.S. Bianchi, On structure constants with two spinning twist-two operators, JHEP 04 (2019) 059 [arXiv: 1901.00679] [INSPIRE].

[27] V.A. Smirnov, Applied asymptotic expansions in momenta and masses, Springer Tracts Mod. Phys. 177 (2002) 1 [INSPIRE]

[28] V.N. Velizhanin, The non-planar contribution to the four-loop universal anomalous dimension in $N=4$ supersymmetric Yang-Mills theory, JETP Lett. 89 (2009) 593 [arXiv: 0902 .4646] [INSPIRE].

[29] V.N. Velizhanin, The non-planar contribution to the four-loop anomalous dimension of twist-2 operators: first moments in $N=4 S Y M$ and non-singlet $Q C D$, Nucl. Phys. B 846 (2011) 137 [arXiv: 1008.2752] [INSPIRE].

[30] V.N. Velizhanin, Non-planar anomalous dimension of twist-2 operators: higher moments at four loops, Nucl. Phys. B 885 (2014) 772 [arXiv:1404.7107] [INSPIRE].

[31] Yu. L. Dokshitzer, G. Marchesini and G.P. Salam, Revisiting parton evolution and the large- $x$ limit, Phys. Lett. B 634 (2006) 504 [hep-ph/0511302] [INSPIRE].

[32] N. Gromov, V. Kazakov, S. Leurent and D. Volin, Quantum spectral curve for planar $N=4$ super-Yang-Mills theory, Phys. Rev. Lett. 112 (2014) 011602 [arXiv:1305.1939] [INSPIRE].

[33] S. Giombi and S. Komatsu, More exact results in the Wilson loop defect CFT: bulk-defect OPE, nonplanar corrections and quantum spectral curve, J. Phys. A 52 (2019) 125401 [arXiv: 1811.02369] [INSPIRE].

[34] R.H. Boels, T. Huber and G. Yang, Four-loop nonplanar cusp anomalous dimension in $N=4$ supersymmetric Yang-Mills theory, Phys. Rev. Lett. 119 (2017) 201601 [arXiv: 1705.03444] [INSPIRE].

[35] R.H. Boels, T. Huber and G. Yang, The Sudakov form factor at four loops in maximal super Yang-Mills theory, JHEP 01 (2018) 153 [arXiv:1711.08449] [INSPIRE].

[36] R.H. Boels, T. Huber and G. Yang, The nonplanar cusp and collinear anomalous dimension at four loops in $N=4 S Y M$ theory, PoS (RADCOR2017)042 (2017) [arXiv:1712.07563] [INSPIRE].

[37] B. Basso, S. Komatsu and P. Vieira, Structure constants and integrable bootstrap in planar $N=4 S Y M$ theory, arXiv:1505.06745 [INSPIRE].

[38] T. Fleury and S. Komatsu, Hexagonalization of correlation functions, JHEP 01 (2017) 130 [arXiv: 1611.05577] [INSPIRE].

[39] B. Eden and A. Sfondrini, Tessellating cushions: four-point functions in $N=4 S Y M$, JHEP 10 (2017) 098 [arXiv: 1611.05436] [INSPIRE]. 
[40] T. Bargheer, J. Caetano, T. Fleury, S. Komatsu and P. Vieira, Handling handles: nonplanar integrability in $N=4$ supersymmetric Yang-Mills theory, Phys. Rev. Lett. 121 (2018) 231602 [arXiv: 1711.05326] [INSPIRE].

[41] T. Bargheer, J. Caetano, T. Fleury, S. Komatsu and P. Vieira, Handling handles. Part II. Stratification and data analysis, JHEP 11 (2018) 095 [arXiv: 1809.09145] [INSPIRE].

[42] B. Eden, Y. Jiang, D. le Plat and A. Sfondrini, Colour-dressed hexagon tessellations for correlation functions and non-planar corrections, JHEP 02 (2018) 170 [arXiv:1710.10212] [INSPIRE].

[43] T. Bargheer, F. Coronado and P. Vieira, Octagons I: combinatorics and non-planar resummations, JHEP 08 (2019) 162 [arXiv: 1904.00965] [INSPIRE].

[44] B. Eden, A.C. Petkou, C. Schubert and E. Sokatchev, Partial nonrenormalization of the stress tensor four point function in N=4SYM and AdS/CFT, Nucl. Phys. B 607 (2001) 191 [hep-th/0009106] [INSPIRE].

[45] D. Chicherin et al., Correlation functions of the chiral stress-tensor multiplet in $N=4 S Y M$, JHEP 06 (2015) 198 [arXiv:1412.8718] [INSPIRE].

[46] SageMath webpage, http://www.sagemath.org.

[47] R. Penrose, Twistor algebra, J. Math. Phys. 8 (1967) 345 [inSPIRE].

[48] A. Ferber, Supertwistors and conformal supersymmetry, Nucl. Phys. B 132 (1978) 55 [INSPIRE].

[49] N. Berkovits, Ten-dimensional super-twistors and super-Yang-Mills, JHEP 04 (2010) 067 [arXiv:0910.1684] [INSPIRE].

[50] M.R. Bullimore, Scattering amplitudes and Wilson loops in twistor space, Springer International Publishing, New York, NY, U.S.A. (2014) [INSPIRE].

[51] E. Witten, Perturbative gauge theory as a string theory in twistor space, Commun. Math. Phys. 252 (2004) 189 [hep-th/0312171] [INSPIRE].

[52] R. Boels, L.J. Mason and D. Skinner, Supersymmetric gauge theories in twistor space, JHEP 02 (2007) 014 [hep-th/0604040] [INSPIRE].

[53] B. Eden, G.P. Korchemsky and E. Sokatchev, From correlation functions to scattering amplitudes, JHEP 12 (2011) 002 [arXiv: 1007.3246] [INSPIRE].

[54] S. Coleman, Aspects of symmetry: selected Erice lectures, Cambridge University Press, Cambridge, U.K. (1985) [INSPIRE].

[55] G. Gabadadze and M. Shifman, QCD vacuum and axions: what's happening?, Int. J. Mod. Phys. A 17 (2002) 3689 [hep-ph/0206123] [INSPIRE].

[56] N.I. Usyukina and A.I. Davydychev, Exact results for three and four point ladder diagrams with an arbitrary number of rungs, Phys. Lett. B 305 (1993) 136 [INSPIRE].

[57] B. Eden and V.A. Smirnov, Evaluating four-loop conformal Feynman integrals by D-dimensional differential equations, JHEP 10 (2016) 115 [arXiv:1607.06427] [INSPIRE].

[58] M. Beneke and V.A. Smirnov, Asymptotic expansion of Feynman integrals near threshold, Nucl. Phys. B 522 (1998) 321 [hep-ph/9711391] [INSPIRE].

[59] R.N. Lee, LiteRed 1.4: a powerful tool for reduction of multiloop integrals, J. Phys. Conf. Ser. 523 (2014) 012059 [arXiv: 1310.1145] [INSPIRE]. 
[60] A.V. Smirnov and F.S. Chuharev, FIRE6: Feynman Integral REduction with modular arithmetic, arXiv:1901.07808 [INSPIRE].

[61] R.N. Lee, A.V. Smirnov and V.A. Smirnov, Master integrals for four-loop massless propagators up to transcendentality weight twelve, Nucl. Phys. B 856 (2012) 95 [arXiv:1108.0732] [INSPIRE].

[62] D. Chicherin, J. Drummond, P. Heslop and E. Sokatchev, All three-loop four-point correlators of half-BPS operators in planar $N=4$ SYM, JHEP 08 (2016) 053 [arXiv: 1512.02926] [INSPIRE].

[63] D. Chicherin, A. Georgoudis, V. Gonçalves and R. Pereira, All five-loop planar four-point functions of half-BPS operators in $N=4$ SYM, JHEP 11 (2018) 069 [arXiv:1809.00551] [INSPIRE].

[64] G. Arutyunov and E. Sokatchev, On a large $N$ degeneracy in $N=4 S Y M$ and the AdS/CFT correspondence, Nucl. Phys. B 663 (2003) 163 [hep-th/0301058] [InSPIRE].

[65] G. Arutyunov, S. Penati, A. Santambrogio and E. Sokatchev, Four point correlators of BPS operators in $N=4 S Y M$ at order $g^{4}$, Nucl. Phys. B 670 (2003) 103 [hep-th/0305060] [INSPIRE].

[66] L. Koster, V. Mitev, M. Staudacher and M. Wilhelm, Composite operators in the twistor formulation of $N=4$ supersymmetric Yang-Mills theory, Phys. Rev. Lett. 117 (2016) 011601 [arXiv: 1603.04471] [INSPIRE].

[67] D. Chicherin and E. Sokatchev, Demystifying the twistor construction of composite operators in $N=4$ super-Yang-Mills theory, J. Phys. A 50 (2017) 205402 [arXiv:1603.08478] [INSPIRE].

[68] L. Koster, V. Mitev, M. Staudacher and M. Wilhelm, On form factors and correlation functions in twistor space, JHEP 03 (2017) 131 [arXiv:1611.08599] [INSPIRE]. 